\title{
Evaluation for Adjustment Method of Vehicle's Location by Recognizing Crosswalks
}

\author{
Yoshihisa Yamaguchi ${ }^{1}$, Takashi Nakagawa ${ }^{1}$, Hirokazu Kato ${ }^{2}$, and Shogo Nishida ${ }^{3}$ \\ ${ }^{1}$ Advanced Technology R\&D Center, Mitsubishi Electric Corporation, \\ 8-1-1, Tsukaguchi-honnmachi, Amagasaki, Hyogo, 661-8661, Japan \\ \{Yamaguchi.Yoshihisa@dp, \\ Nakagawa.Takashi@dy\}.MitsubishiElectric.co.jp \\ ${ }^{2}$ Graduate School of Information Science, Nara Institute of Science and Technology \\ kato@is.naist.jp \\ ${ }^{3}$ Graduate School of Engineering Science, Osaka University, \\ 1-3, Machikaneyama, Toyonaka, Osaka, 560-8531, Japan \\ nishidalsys.es.osaka-u.ac.jp
}

\begin{abstract}
We have developed AR-Navi, an in-vehicle navigation system that captures real-time video via an in-vehicle camera and overlays guidance on a video display. In this study, we conducted a driving experiment to evaluate the location-adjustment-method of AR-Navi. We also discuss the registration accuracy of the route guidance that AR-Navi gives at intersections.
\end{abstract}

Keywords: Augmented reality, In-vehicle navigation systems, Route guidance.

\section{Introduction}

In recent years, over 32 million in-vehicle navigation systems have been produced in Japan [1], and have become popular. Their basic functions, such as map indication and route planning, perform sufficiently well, so manufacturers have been mainly improving their user interface design. For example, newer functions are being provided, such as using 3D computer graphics (CG) and mixing aerial photos and existing maps to show cityscapes.

To improve the user interface design, some researchers have developed in-vehicle navigation systems that use video-based augmented reality technology [2-4]. The system has an in-vehicle camera that captures real-time video, and the system then overlays guidance on to the video. Because the video is almost the same as what the driver is seeing, the system provides easily understandable guidance. However, the system has some problems: if registration gaps between real-time video and CG occur driver may be confused. Sawano [2] describes extracting geometry information of roads and drawing $\mathrm{CG}$ roads on to real-time video. $\mathrm{Hu}$ [3] describes extracting future points of roads and adjusting the location between the guidance, which is drawings of the roads, and real-time video. These works detail the high-precision alignment between real-time video and CG with image analysis. The image analysis technology used in these works, however, also has some problems. First, the technology needs 
much time complexity. Second, it is not robust enough for conditions such as rain, night, backlight, and so on, so it is not used in current in-vehicle navigation systems. Therefore, we have developed a registration adjustment method base on recognizing crosswalks and have tried to develop a commercializable system using this method, which needs only a camera and can adapt to various environmental conditions. We call the system AR-Navi.

In this paper, we explain the adjustment method used for AR-Navi. Then we detail the evaluation of the method by driving on public roads and discuss the registration accuracy of the route guidance that AR-Navi shows at intersections.

\section{Registration Adjustment Based on Recognizing Crosswalks}

\subsection{Location Gap}

When AR-Navi overlays information on to the video, it adjusts positions between the information and the video on the basis of the location and direction of the driver's vehicle. If the location and direction have errors, gaps occur in the overlaid image.

Almost all current in-vehicle navigation systems calculate their own location and direction on the basis of two steps [5]. At the first step, the systems estimate their location and direction using a combination of Global Positioning System (GPS) data and autonomous navigation data such as that from gyros and speed sensors. At the second step, they use a map matching method, which compares the result of the first step with road shapes. Furthermore, the systems correct the vehicle location if the driving greatly changes (such as at turns) or there are differences between the driving and road shapes. Therefore, immediately after a vehicle turns, the location and direction changes highly accurately. However, huge errors sometimes happen if a vehicle goes straight for a long time without enough GPS signal. We have divided the gaps between location and direction into four categories and consider solutions as follows.

- Back-and-forth gap: As I have indicated before, the longer a vehicle goes straight, the more back-and-forth gap widens. Because a huge back-and-forth gap makes drivers confuse a wrong intersection with the right one, it is necessary to narrow this gap.

- Right-and-left gap: Because of using map matching, the calculated location rarely leaves the road. Therefore, the right-and-left gap does not exceed road width. If it does not depart from the road, AR-Navi does not give wrong guidance, so this gap is not a practical issue.

- Rotation gap: Current in-vehicle navigation systems estimate their direction on the basis of gyros. The rotation gap widens easily because gyros integrate the change in angle. Moreover, the measurement is delayed. It misaligns guidance and video when the vehicle direction changes greatly, such as when making a U-turn. Thus, it is necessary to narrow the rotation gap.

- Pitch-angle gap: If a vehicle goes along at a constant speed, the vehicle is parallel to the ground, but while accelerating or braking the vehicle inclines. The pitchangle gap, however, is not a practical problem because it is only a temporary phenomenon. 
Back-and-forth and rotation gaps must be adjusted when in-vehicle systems overlay $\mathrm{CG}$ information on to the video. Because we use an image-filtering method to remove images that have large rotation gaps [4], we only have to deal with back-andforth gaps. To resolve this problem, we propose registration adjustment based on recognizing crosswalks.

\subsection{Proposed Method}

To decrease back-and-forth gaps, we propose adjusting the location at intersections, which are found using image analysis, corresponding with intersections on the maps. It takes the following conditions to use the algorithm in current in-vehicle navigation systems.

- Little time complexity: For the algorithm to work in current in-vehicle navigation systems, time complexity needs to be small.

- High robustness: The algorithm has to be robust to work in different weather and levels of brightness.

Considering the above conditions, this method finds crosswalks by analyzing only one line that is set on a captured image and located a certain distance ahead of the driver's vehicle. The line is called the scan line.

Then it calculates the back-and-forth gap by comparing the found crosswalk with the corresponding crosswalk on the map, because it is assumed that there is an intersection between a pair of crosswalks.

This method has the following merits.

- It needs little time complexity because only the image of a narrow area, the scan line, is analyzed.

- If the scan line is set near enough, it is rarely interfered with by other vehicles while driving, so image analysis is stable.

- If environmental conditions are unfavorable, such as rain and darkness, the scan line is lit by the car's headlights.

This method works as follows.

1. It judges whether a zebra pattern exists or not.

2. It stores the position where vehicle goes into and leaves out of the zebra pattern.

3. It judges whether the zebra pattern is a crosswalk or not.

4. It recognizes a pair of crosswalks.

5. It calculates the distance between the two crosswalks.

6. It calculates midway between a location going into and location coming out of a crosswalk, if the distance is not too short or too long.

7. It adjusts the back-and-forth gap by difference between the calculated midway and the corresponding crosswalks on the map.

Judging whether a Crosswalk Exists or not. To find crosswalks, a crosswalk empathies filter is applied to the brightness of each pixel on the scan line. Fig. 1 shows the crosswalk empathies filter. It is easy to obtain high-accuracy binarized results because the filter turns the raw brightness into the brightness shown in Fig. 2. 
Then this method judges whether a crosswalk exists or not on the scan line by checking the number of black and white runs. If the lengths of each run are almost same as the widths of the white lines of a crosswalk in the image and the black and white runs alternate, this method judges it to be a crosswalk.

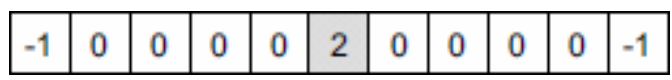

Fig. 1. Crosswalk empathies filter

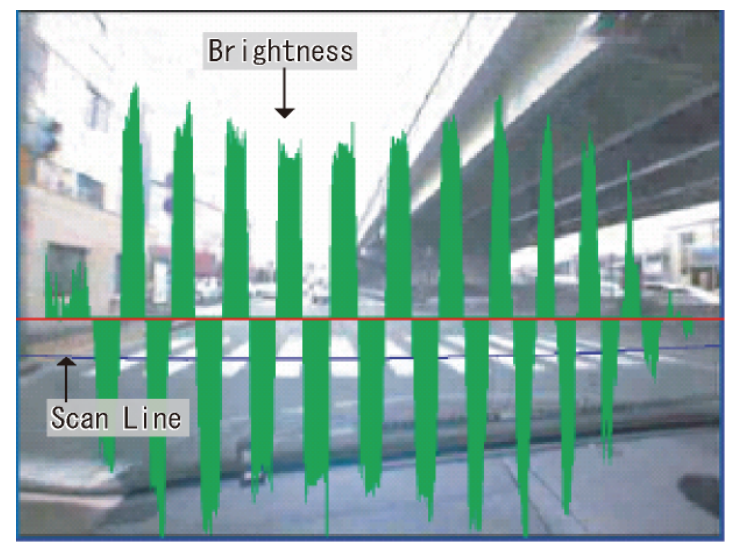

Fig. 2. Result of applying of Crosswalk empathies filter

Judging whether a Pair of Crosswalks is Appropriate or not. As was discussed in section 2.2, to find a crosswalk, two crosswalks must be found. Even if two crosswalks are found, however, there is a possibility of image analysis failing. For this reason, this method does not adjust location if the width of a crosswalk or the distance between the two crosswalks is too short or too long.

\section{Evaluation of Proposed method}

\subsection{Conditions}

We installed AR-Navi based on our in-vehicle navigation system and a lens, the specifications of which were $320 * 240$ pixels, $6 \mathrm{~mm}$ focal length, and 47-degree angle of view. We drove in urban areas using AR-Navi and measured the back-andforth gaps.

When registration adjustments not caused by the proposed method occurred, the effect of the proposed method was not measured. We set the following conditions:

- No turning: After a vehicle turns, its location is adjusted by the map matching method. Therefore, we needed to drive straight and not turn.

- No using GPS: General in-vehicle navigation systems adjust the vehicle's location if there is huge difference between the location based on the GPS signal and one recognized by the systems. Therefore, we needed to remove the GPS. 
We selected a road that had four lanes and ran from north to south for about 4.6 kilometers after considering the above reasons. We drove along the road with AR-Navi and counted the number of times that the proposed method adjusted registration, and we measured the adjustment length to examine the effectiveness of the proposed method. During this driving experiment, AR-Navi was not connected to a GPS receiver.

\subsection{Discussion}

Adjustment lengths are shown in Table 1. The proposed method was carried out nine times and lengths adjusted an average of 6.1 meters in the direction traveled. Fig. 3 shows the relationship between the adjustment length and distance from the adjusted point to the previous adjusted point. (The first adjusted point is not plotted in Fig. 3 because it has no previous point.) The dashed line of Fig. 3 shows straight-line approximation. The correlation coefficient between the vertical axis and the horizontal

Table 1. Adjustment lengths of the proposed method

\begin{tabular}{|c|}
\hline Length [m] \\
\hline 10.6 \\
\hline 3.2 \\
\hline 9.4 \\
\hline 5.4 \\
\hline 4.9 \\
\hline 2.6 \\
\hline 3.7 \\
\hline 8.1 \\
\hline 6.8 \\
\hline
\end{tabular}

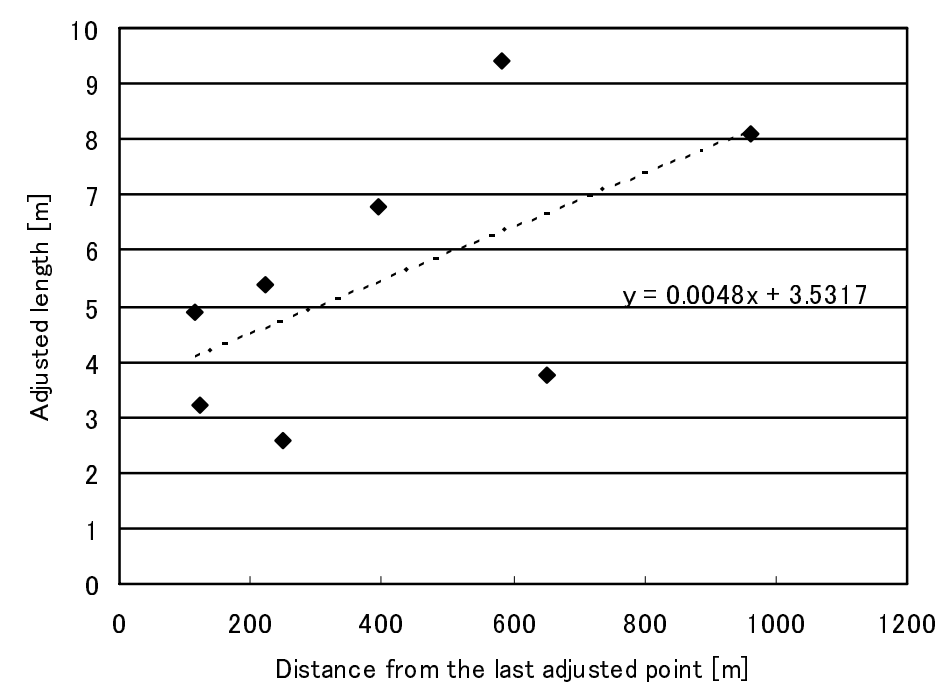

Fig. 3. Relationship between adjustment length and distance to the last adjusted point 
axis is 0.59 . This shows that the adjustment length decreased along with distance from the last adjustment point. Thus, we presume that the back-and-forth gap disappears immediately after the registration adjustment occurs but the gap widens with driving.

Fig. 3 shows the vehicle's location is adjusted by several meters even when a vehicle drives only 100 meters after registration adjustment. Therefore, the adjustment length includes the following gaps:

1. Random gap

2. Constant gap

3. Growing gap

Random gaps (1) are caused by the frame rate of AR-Navi and the accuracy of the maps. First, AR-Navi uses a 10-frame-rate video because the navigation system has limited update currency. AR-Navi sometimes could not recognize crosswalks correctly. For example, a vehicle runs one meter per frame when it runs at $36 \mathrm{~km} / \mathrm{h}$. Second, maps used in conventional in-vehicle navigation systems are not perfectly accurate; because there are errors in the maps, random gaps occur.

Constant gaps (2) are caused by time lags in carrying out the proposed method. Registration adjustment is sometimes not carried out immediately because many processes are running and some processes, such as drawing maps, have higher priority. Because a vehicle runs while its location is being adjusted, differences come about between the adjusted length and actual necessary length, that is, the next adjustment length increases. According to the y-intercept of dashed line in Fig. 3, the constant gap is 3.5 meters. Because this gap can be removed by modifying programs, we discuss the following without reference to constant gaps.

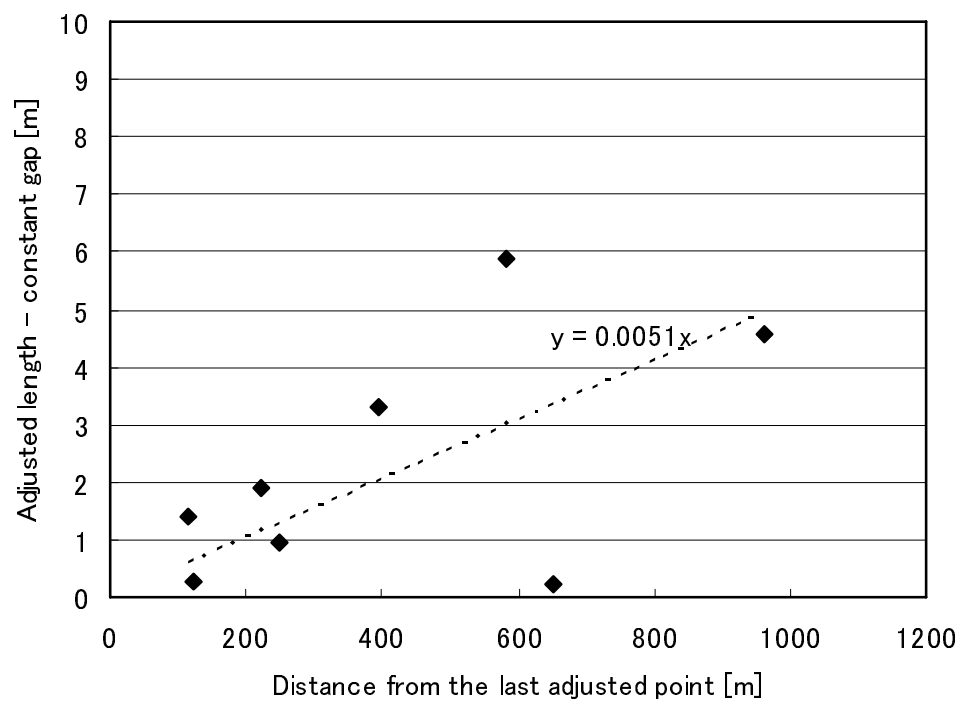

Fig. 4. Relationship between growing gap and distance to the last adjusted point 
Growing gaps (3) are caused by autonomous navigation. To clear the growing gap, Fig. 4 shows the relationship between the adjustment length minus the constant gap (3.5 meters) and distance from the current adjusted point to the previous adjusted point. The average size of a growing gap is 2.3 meters and the maximum growing gap's size is less than 6 meters. The dashed line in Fig. 4 shows straight-line approximation and represents the gap-growing model. To examine the model, we drove on the same roads as the above experiment without using the proposed method and measured the back-and-forth gap between the actual and estimated locations using an in-vehicle navigation system. The measured gap was 20 meters after driving 4.6 kilometers. On the other hand, the theoretical gap is 23.5 meters according to the dashed line. Because these two gaps are quite different, the model is correct. The back-and-forth gap of AR-Navi increases by 0.51 meters every 100 meters.

In the next section, we discuss the accuracy of route guidance of AR-Navi on the basis of commercialization.

\section{Discussion about Accuracy of Route Guidance}

If route guidance information is overlaid on an intersection area in a real-time video, the driver recognizes the intersection where he or she will turn (hereafter the target intersection). The conventional in-vehicle navigation systems do not display roads that are less than 5.5 meters wide except when vehicles are close to their destinations. Therefore, we discuss whether AR-Navi can overlay information on 5.5-meter wide intersections. This is the most taxing of conditions because intersections are normally wider than 5.5 meters. To overlay information on a 5.5-meter intersection, we calculated the probability that the proposed method limited the back-and-forth gap to 5.5 meters. In the following discussion, only growing gaps are considered because random gaps do not occur on average and constant gaps can be removed.

We model the probability that the proposed method recognizes a pair of crosswalks and adjusts the vehicle's location accordingly. Because where crosswalks exist is random natural phenomenon, the probability follows Poisson distribution. Poisson distribution indicates that if the expected number of occurrences in a certain interval is $\lambda$, the probability that they are exactly $\mathrm{k}$ occurrences is equal to formula (1) ( $\mathrm{e}$ is the base of the natural logarithm).

$$
P_{(N=k)}=e^{-\lambda} \lambda^{k} / k !
$$

The limit to which growing gaps do not exceed 2.75 meters is 539.2 meters. Therefore, we used Poisson distribution with a 539.2-meter interval and calculated the probability that the vehicle's location is adjusted at least once per an interval. $\lambda$, that is the expected number of times registration adjustment occurs in 539.2 meters, is calculated as formula (2), because registration adjustment operates once every 412.6 meters on average according to the experiment.

$$
\lambda=539.2 / 412.6 \approx 1.31
$$


Also, the probability that registration adjustment does not operate is equal to formula (3).

$$
P_{(N=0)}=0.270 \cdots \approx 0.27
$$

In other words, the probability that back-and-forth gaps do not exceed 2.75 meters and AR-Navi correctly overlays information on an intersection is 73 percent by using the proposed method even in the most demanding of conditions.

We have evaluated in the most difficult conditions, which were based on the narrowest roads. Nevertheless, considering average roads, it is possible that AR-Navi overlay information on an intersection if back-and-forth gaps are greater than 2.75 meters. Moreover, because AR-Navi can estimate back-and-forth gaps, AR-Navi can switch information displayed in accordance with back-and-forth gaps and the intersections' sizes. For example, conventional CG guidance is displayed if the estimated gap exceeds the size of the target intersection. Therefore, even if a back-and-forth gap is big, AR-Navi can still display correct route guidance.

In addition, considering general situations, there is less probability that the gap exceeds 2.75 meters at the target intersection because of following reasons.

- Frequency of turning

- Guidance timing

First, it is unusual that a vehicle would go straight on local roads without GPS signal for long distances as we did in the experiment. The vehicle's location is frequently adjusted in general situations because turnings trigger registration adjustment. Furthermore, if there is a big gap between the vehicle's location in the real world and that on its in-vehicle navigation system, the system adjusts the location via the GPS signal. Therefore, back-and-forth gaps occur less than in the experimental results.

Second, guidance is not always displayed if the vehicle's location is not adjusted for long distances and the gap becomes bigger. If AR-Navi recognizes a pair of crosswalks before reaching the target intersection, AR-Navi adjusts the vehicle's location and the gap disappears.

For the above reasons, the probability that AR-Navi cannot overlay CG information on the target intersection is less than 27 percent in general driving. Therefore, AR-Navi gives exact guidance practically thanks to the proposed method.

\section{Conclusion}

In this paper, we conducted a driving experiment to evaluate the registration adjustment based on recognizing crosswalks and showed that it occurs once every 412.6 meters and adjusts 2.3 meters on average. In addition, we discussed the accuracy of route guidance when using the proposed method and showed AR-Navi can display exact and easy-to-understand guidance. An evaluation of AR-Navi's usability should be a future work. 


\section{References}

1. Japanese Ministry of Land, Infrastructure, Transport and Tourism, Road Bureau ITS Homepage,

http://www.mlit.go.jp/road/ITS/j-html/vics_pdf/navi_vics.pdf

2. Sawano, H., Okada, M.: A Study on an Augmented Reality-based Rendering Method for Car Navigation Utilizing Real-time Video. In: FCV 2004 - Tenth Korea-Japan Joint Workshop on Frontiers of Computer Vision, Fukuoka Japan (February 2004)

3. Hu, Z., Uchida, K.: Solution of Camera Registration Problem via 3D-2D Parameterized Model Matching for On-Road Navigation. International Journal of Image and Graphics 4(1), 3-20 (2004)

4. Yamaguchi, Y., Nakagawa, T., Akaho, K., Honda, M., Kato, H., Nishida, S.: AR-Navi: an in-vehicle navigation system using video-based augmented reality technology. In: Proceedings of the 12th International Conference on Human-Computer Interaction, pp. 1139-1147 (2007)

5. Quddus, M.A., Ochieng, W.Y., Zhao, L., Noland, R.B.: A General Map Matching Algorithm for Transport Telematics Applications. The GPS Solutions Journal 7(3), 157-167 (2003) 